\title{
Synthesis and characterization of the tin iodide borate $\mathrm{Sn}_{3}\left[\mathbf{B}_{3} \mathbf{O}_{7}\right] \mathbf{I}$
}

Sandra Schönegger ${ }^{\text {a }}$, Florian Pielnhofer ${ }^{b}$, Andreas Saxer ${ }^{\mathrm{c}}$, Klaus Wurst ${ }^{\mathrm{a}}$, and Hubert Huppertz ${ }^{a}$,*

\footnotetext{
a Institut für Allgemeine, Anorganische und Theoretische Chemie, Universität Innsbruck, Innrain 80-82, 6020 Innsbruck, Austria

b Institut für Anorganische Chemie, Universität Regensburg Universitätsstraße 31, D-93053 Regensburg

c Institut für Konstruktion und Materialwissenschaften, Universität Innsbruck, Technikerstraße 13, A-6020 Innsbruck
} 


\section{Supporting Information}

Table S1. Wyckoff positions, atomic coordinates, and equivalent isotropic displacement parameters $U_{e q} /$ $\AA^{2}$ of $\mathrm{Sn}_{3}\left[\mathrm{~B}_{3} \mathrm{O}_{7}\right]$ I. $U_{e q}$ is defined as one third of the trace of the orthogonalized $U_{i j}$ tensor (standard deviations in parentheses).

Table S2. Anisotropic displacement parameters $U_{i j}$ in $\AA^{2}$ (standard deviations in parentheses).

Table S3. Interatomic distances in pm (standard deviations in parentheses).

Table S4. Bond angles in deg (standard deviations in parentheses).

Table S5. Comparison of the single-crystal lattice parameters of $\mathrm{Sn}_{3}\left[\mathrm{~B}_{3} \mathrm{O}_{7}\right] \mathrm{I}$ and $\mathrm{Sn}_{3}\left[\mathrm{~B}_{3} \mathrm{O}_{7}\right] \mathrm{F}$.

Table S6: DFT optimized lattice parameters and band gaps of $\operatorname{Sn}_{3}\left[\mathrm{~B}_{3} \mathrm{O}_{7}\right] \mathrm{I}$ and $\mathrm{Sn}_{3}\left[\mathrm{~B}_{3} \mathrm{O}_{7}\right] \mathrm{F}$. 
Table S1. Wyckoff positions, atomic coordinates, and equivalent isotropic displacement parameters $U_{\text {eq }}$ $\AA^{2}$ of $\mathrm{Sn}_{3}\left[\mathrm{~B}_{3} \mathrm{O}_{7}\right]$ I. $U_{\text {eq }}$ is defined as one third of the trace of the orthogonalized $U_{\mathrm{ij}}$ tensor (standard deviations in parentheses).

\begin{tabular}{llllll}
\hline Atom & Wyckoff Position & $x$ & $y$ & $z$ & $U_{\text {eq }}$ \\
\hline Sn1 & $8 c$ & $0.35986(3)$ & $0.90782(4)$ & $0.32630(2)$ & $0.00927(7)$ \\
Sn2 & $8 c$ & $0.42444(4)$ & $0.68058(5)$ & $0.48484(2)$ & $0.02364(9)$ \\
Sn3 & $8 c$ & $0.20719(3)$ & $0.67463(4)$ & $0.19049(2)$ & $0.00967(7)$ \\
I1 & $8 c$ & $0.18290(5)$ & $0.56430(7)$ & $0.53226(4)$ & $0.0475(2)$ \\
O1 & $8 c$ & $0.3821(3)$ & $0.1314(4)$ & $0.2867(2)$ & $0.0097(5)$ \\
O2 & $8 c$ & $0.4872(3)$ & $0.3564(4)$ & $0.2440(2)$ & $0.0084(5)$ \\
O3 & $8 c$ & $0.2644(3)$ & $0.4000(4)$ & $0.2432(2)$ & $0.0088(5)$ \\
O4 & $8 c$ & $0.3889(3)$ & $0.4928(4)$ & $0.3358(2)$ & $0.0118(6)$ \\
O5 & $8 c$ & $0.3933(3)$ & $0.6210(4)$ & $0.2302(2)$ & $0.0101(5)$ \\
O6 & $8 c$ & $0.5264(3)$ & $0.2757(4)$ & $0.3569(2)$ & $0.0123(6)$ \\
O7 & $8 c$ & $0.4750(4)$ & $0.4529(5)$ & $0.4419(2)$ & $0.0223(8)$ \\
B1 & $8 c$ & $0.3869(4)$ & $0.4666(6)$ & $0.2635(2)$ & $0.0074(7)$ \\
B2 & $8 c$ & $0.4633(5)$ & $0.4066(6)$ & $0.3774(3)$ & $0.0120(8)$ \\
B3 & $8 c$ & $0.4992(4)$ & $0.2171(5)$ & $0.2905(2)$ & $0.0069(7)$ \\
\hline
\end{tabular}

Table S2. Anisotropic displacement parameters $U_{\mathrm{ij}}$ in $\AA^{2}$ (standard deviations in parentheses).

\begin{tabular}{lllllll}
\hline Atom & $U_{11}$ & $U_{22}$ & $U_{33}$ & $U_{23}$ & $U_{13}$ & $U_{12}$ \\
\hline Sn1 & $0.0086(1)$ & $0.0085(2)$ & $0.0107(2)$ & $0.00251(9)$ & $-0.00001(9)$ & $0.00021(9)$ \\
Sn2 & $0.0341(2)$ & $0.0351(3)$ & $0.0733(4)$ & $-0.0141(3)$ & $-0.0070(3)$ & $-0.0009(2)$ \\
Sn3 & $0.0318(2)$ & $0.0176(2)$ & $0.0215(2)$ & $-0.0010(2)$ & $-0.0063(2)$ & $0.0086(2)$ \\
I1 & $0.0077(1)$ & $0.0010(2)$ & $0.0114(2)$ & $-0.00098(9)$ & $-0.00150(9)$ & $0.00091(9)$ \\
O1 & $0.005(1)$ & $0.008(2)$ & $0.016(2)$ & $0.001(1)$ & $-0.002(1)$ & $-0.000(2)$ \\
O2 & $0.007(1)$ & $0.004(1)$ & $0.014(2)$ & $0.001(1)$ & $0.002(1)$ & $0.0005(9)$ \\
O3 & $0.005(1)$ & $0.007(2)$ & $0.014(2)$ & $-0.001(1)$ & $0.001(1)$ & $-0.0013(9)$ \\
O4 & $0.012(2)$ & $0.011(2)$ & $0.013(2)$ & $-0.001(1)$ & $-0.001(1)$ & $0.003(1)$ \\
O5 & $0.005(1)$ & $0.005(2)$ & $0.020(2)$ & $0.002(1)$ & $-0.000(1)$ & $-0.0006(9)$ \\
O6 & $0.016(2)$ & $0.011(2)$ & $0.010(2)$ & $-0.003(1)$ & $-0.005(1)$ & $0.004(1)$ \\
O7 & $0.030(2)$ & $0.023(2)$ & $0.014(2)$ & $-0.007(2)$ & $-0.009(2)$ & $0.009(2)$ \\
B1 & $0.006(2)$ & $0.006(2)$ & $0.010(2)$ & $-0.001(2)$ & $-0.001(2)$ & $-0.000(2)$ \\
B2 & $0.011(2)$ & $0.014(2)$ & $0.012(2)$ & $-0.003(2)$ & $-0.002(2)$ & $0.001(2)$ \\
B3 & $0.005(2)$ & $0.006(2)$ & $0.010(2)$ & $-0.001(2)$ & $-0.001(2)$ & $0.000(2)$ \\
\hline
\end{tabular}


Table S3. Interatomic distances in pm (standard deviations in parentheses).

\begin{tabular}{|c|c|c|c|}
\hline Sn1 O1 & 208.1(3) & $\mathrm{B} 1 \mathrm{O} 2$ & $148.2(6)$ \\
\hline $\mathrm{Sn} 1 \quad \mathrm{O} 3^{1}$ & 214.1(3) & B1 O3 & $148.8(5)$ \\
\hline $\mathrm{Sn} 1 \mathrm{O} 2^{2}$ & 221.2(3) & B1 O4 & $147.5(6)$ \\
\hline \multirow[t]{2}{*}{ Sn1 O4 } & 277.1(3) & B1 O5 & $147.9(6)$ \\
\hline & $\varnothing=230.1$ & & $\varnothing=146.8$ \\
\hline Sn2 I1 & 293.3(1) & B3 O1 & $145.4(5)$ \\
\hline \multirow[t]{3}{*}{ Sn2 O7 } & 219.4(4) & B3 O2 & $151.9(6)$ \\
\hline & $\varnothing=256.4$ & B3 O5 & $147.4(5)$ \\
\hline & & B3 O6 & $145.7(6)$ \\
\hline $\mathrm{Sn} 3 \mathrm{O}^{4}$ & 219.4(3) & & $\varnothing=146.8$ \\
\hline $\mathrm{Sn} 3 \mathrm{O}^{4}$ & 221.7(3) & & \\
\hline Sn3 O5 & 219.7(3) & B2 O4 & 137.1(6) \\
\hline $\mathrm{Sn} 3 \mathrm{O} 2$ & 281.0(3) & B2 O6 & $136.9(6)$ \\
\hline \multirow[t]{2}{*}{ Sn3 I1 } & $389.9(3)$ & B2 O7 & $136.5(6)$ \\
\hline & $\varnothing=246.3$ & & $\varnothing=146.8$ \\
\hline
\end{tabular}

Table S4. Bond angles in deg (standard deviations in parentheses).

\begin{tabular}{|c|c|c|c|c|}
\hline & Sn1 O3 & $78.4(2)$ & $\mathrm{O} 4 \mathrm{~B} 1 \mathrm{O} 2$ & $110.4(3)$ \\
\hline $\mathrm{O} 1$ & Sn1 O111 & $81.5(2)$ & O4 B1 O5 & $108.2(3)$ \\
\hline $\mathrm{O} 3$ & Sn1 O2 & $87.4(2)$ & $\mathrm{O} 2 \mathrm{~B} 1 \mathrm{O} 5$ & $114.2(3)$ \\
\hline \multirow[t]{3}{*}{$\mathrm{O} 1$} & Sn1 O4 & 84.1(1) & O4 B1 O3 & $110.1(3)$ \\
\hline & & $\varnothing=82.9$ & $\mathrm{O} 2 \mathrm{~B} 1 \mathrm{O} 3$ & 109.1(3) \\
\hline & & & O5 B1 O3 & $104.8(3)$ \\
\hline O7 & Sn2 I1 & $92.3(2)$ & & $\varnothing=109.5$ \\
\hline $\mathrm{O} 1$ & Sn3 O5 & $92.2(2)$ & O1 B3 O6 & $113.0(4)$ \\
\hline $\mathrm{O} 1$ & Sn3 O3 & $77.3(2)$ & O1 B3 O5 & $112.6(3)$ \\
\hline O5 & $\mathrm{Sn} 3 \mathrm{O} 3$ & $83.1(2)$ & O6 B3 O5 & $107.0(3)$ \\
\hline \multirow[t]{8}{*}{$\mathrm{O} 2$} & Sn3 O3 & $55.9(1)$ & $\mathrm{O} 1 \mathrm{~B} 3 \mathrm{O} 2$ & $106.6(3)$ \\
\hline & & $\varnothing=77.1$ & O6 B3 O2 & $108.5(3)$ \\
\hline & & & $\mathrm{O} 5 \mathrm{~B} 3 \mathrm{O} 2$ & $109.0(3)$ \\
\hline & & & & $\varnothing=109.5$ \\
\hline & & & O7 B2 O6 & $118.5(4)$ \\
\hline & & & $\mathrm{O} 7 \mathrm{~B} 2 \mathrm{O} 4$ & $118.6(4)$ \\
\hline & & & O6 B2 O4 & $122.8(4)$ \\
\hline & & & & $\varnothing=120.0$ \\
\hline
\end{tabular}


Table S5: Comparison of the single-crystal lattice parameters of $\mathrm{Sn}_{3}\left[\mathrm{~B}_{3} \mathrm{O}_{7}\right] \mathrm{I}$ and $\mathrm{Sn}_{3}\left[\mathrm{~B}_{3} \mathrm{O}_{7}\right] \mathrm{F}$.

\begin{tabular}{|c|c|c|}
\hline Crystal system & $\begin{array}{l}\mathbf{S n}_{3}\left[\mathbf{B}_{3} \mathbf{O}_{7}\right] \mathbf{I} \\
\text { orthorhombic }\end{array}$ & $\begin{array}{l}\mathbf{S n}_{3}\left[\mathbf{B}_{3} \mathbf{O}_{7}\right] \mathbf{F} \\
\text { orthorhombic }\end{array}$ \\
\hline Space group & $P b c a($ no. 61$)$ & $P n a 2_{1}$ (no. 33) \\
\hline$a, \mathrm{pm}$ & $1071.8(3)$ & $922.38(4)$ \\
\hline$b, \mathrm{pm}$ & $852.3(2)$ & $769.78(4)$ \\
\hline$c, \mathrm{pm}$ & $2016.8(5)$ & $1221.88(6)$ \\
\hline$V, \mathrm{~nm}^{3}$ & $1842.4(8)$ & $867(7)$ \\
\hline Formula units per cell, $Z$ & 8 & 4 \\
\hline
\end{tabular}

Table S6: DFT optimized lattice parameters of $\mathrm{Sn}_{3}\left[\mathrm{~B}_{3} \mathrm{O}_{7}\right] \mathrm{I}$.

\begin{tabular}{lllll}
\hline functional & $a / \mathrm{pm}$ & $b / \mathrm{pm}$ & $c / \mathrm{pm}$ & $V / \mathrm{nm}^{3}$ \\
\hline PBE & 1103.7 & 862.2 & 2058.5 & 19.590 \\
HSE06 & 1088.8 & 857.4 & 2046.1 & 19.102 \\
\hline
\end{tabular}

